\title{
KARTAGENER SYNDROME: A TRIAD OF CHRONIC SINUSITIS, BRONCHIECTASIS AND SITUSINVERSUS
}

Arvinder Singh, Sohan Singh, Manjeet Kaur

1. Associate Professor, Department of Radiodiagnosis, Government Medical College, Amritsar.

2. Professor, Department of Radiodiagnosis, Government Medical College, Amritsar.

3. Associate Professor, Department of Physiology, S. G. R. D, Amritsar.

\section{CORRESPONDING AUTHOR}

Dr. Arvinder Singh,

316-A Moon Avenue Street No.1,

Majitha Road Amritsar Punjab INDIA,

E-mail: arvinderdr@rediffmail.com,

Ph: 00919878655028

ABSTRACT: Kartagener syndrome is an autosomal recessive disorder with a triad of bronchiectasis, recurrent episodes of sinusitis and situs inversus in early life, with reduced fertility in the later part of life. It is a rare genetic disorder of primary ciliary dyskinesia (PCD) with an estimated incidence of 1: 20,000 to 30,000. Here we present an 11 year old male child who presented with bronchiectasis, recurrent episodes of sinusitis and situs inversus which fits into the triad of Kartagener syndrome. Early diagnosis and treatment of this syndrome is important to prevent the deterioration of the lung function and the morbidity caused due to severe intractable upper and lower respiratory tract infections.

KEYWORDS: Bronchiectasis, Situs Inversus, Chronic Sinusitis

CASE REPORT: An 11 year old male child presented with history of recurrent episodes of cough, copious mucoid to mucopurulent sputum, persistent nasal congestion with occasional mucoid nasal discharge. He had a past history of episodes of pneumonias somewhat relieved with antibiotics. No history of similar presentation in his siblings. The total leucocyte count was raised with increased neutrophils and eosinophils. ENT examination showed marked nasal congestion with nasal mucosal hypertrophy. On chest percussion, cardiac dullness was elicited in right precordium. Hepatic dullness was elicited on the left side with a tympanic note on the right side. On auscultation, the heart sounds were normal and heard over the right precordium. Fine crepitations were heard in both lower lung fields.

On chest $\mathrm{X}$ ray, the cardiac apex and aortic knuckle were on right side suggestive of dextrocardia. The hepatic shadow was seen on the left side and gastric fundus on the right side suggestive of situs inversus totalis [Figure 1].

Contrast enhanced CT scan chest showed features of dextrocardia with right sided aortic arch [Figure 2]. Multiple well defined intrapulmonary cystic lesions giving a bunch of grape appearances in the right middle and lower lobes suggestive of central type of bronchiectasis with peribronchial cuffing [Figure 3]. Few of these cystic areas showed air-fluid levels in them indicative of superadded infection. The major vessels like descending aorta and the pulmonary trunk were right sided.

Contrast enhanced CT scan of upper abdomen showed complete situs inversus with Hepatobiliary system on the left side and spleen, gastric fundus and splenic flexure on right side with a raised right dome of diaphragm [Figure 4]. 
Computed tomography of the paranasal sinuses showed mild concentric type of mucosal hypertrophy involving both maxillary antra and bilateral inferior turbinates hypertrophy [Figure 5]. Both osteomeatal complexes were normal and patent.

Pulmonary function tests at the first visit revealed markedly reduced FEV with restrictive pulmonary function tests.

DISCUSSION: Kartagener syndrome is an autosomal recessive disorder with a triad of bronchiectasis, recurrent episodes of sinusitis and situs inversus. It is an entity of primary ciliary dyskinesia with an incidence of 1 in 20000-300001. There are ultra structural defects in the cilia of respiratory mucosa leading to ineffective mucociliary clearance predisposing to recurrent sinopulmonary infections, finally leading to irreversible bronchopulmonary damage. Females can present with subfertility due to sluggish movement of ova in the fallopian tubes, while males demonstrate infertility secondary to immotile spermatozoa. ${ }^{2,3}$ The patient presents with clinical manifestations of bronchiectasis and chronic sinusitis with situs inversus. The importance of diagnosing this rare disorder lies in the fact that deterioration in lung function occurs early in childhood ${ }^{3,4}$.

The triad of bronchiectasis, sinusitis, and situs inversus was first described by Siewert in 1904, although its usual eponym is Kartagener syndrome - after the Swiss pediatrician Manes Kartagener who described 4 cases with similar features in $1933^{3}$. It is associated with mutations in genes, mainly affecting DNAI1 on 17 chromosome 9p21-p13, DNAH5 14 chromosome 5p15p14 and DNAH11 on 18 chromosome 7p21 which results in numerous defects including structural 19 abnormalities of the dynein arms, radial spokes, and microtubules of the cilia ${ }^{5,6,9}$.

The clinical features of PCD have been described in primary ultrastructural defects in cilia and spermatozoa tail. The axoneme is the key component of the cytoskeleton and has characteristic nine plus two arrays of microtubules. The nexin links and spokes seem to provide structural rigidity to the axoneme. Dynein arms extend from one side of a doublet in a clockwise direction, when viewed from the tip of the cilium. They contain most of the ATPase activity of the axoneme and are important in releasing energy for sliding and bending of microtubules and ciliary motion?

The tracheobronchial tree is ciliated to the level of the respiratory bronchioles, each ciliated cell having about 200 cilia. Mucociliary transport in the respiratory tract is important for the normal respiratory function and resistance to respiratory infection ${ }^{8}$. Defect in the cilia presents with a typical clinical picture of PCD i.e. chronic productive cough traced back to early childhood or infancy, chronic rhinitis, chronic or recurrent maxillary sinusitis in childhood. Bronchiectasis is not present at birth, but may develop early, sometimes even in childhood. The most common respiratory pathogens are Haemophilus Influenzae and Streptococcus pneumoniae. Most males are sterile, but many females have a lowered fertility. About $50 \%$ of patients have situs inversus viscerum ${ }^{9}$.

High Resolution CT images help in assessment of severity and scoring of bronchiectasis. Score 0 indicates no evidence of bronchiectasis; score 1 mild bronchiectasis with bronchial dilatation two times the diameter of the accompanying blood vessel, score 2 moderate bronchiectasis with bronchial dilatation two to three times vessel diameter \& score 3 severe bronchiectasis with bronchial dilatation more than three times vessel diameter. The distribution pattern of bronchiectasis can be classified in each lobe as central, peripheral or diffuse with the presence or absence of peribronchial thickening and mucus plugging. 
Kartagener syndrome can have variable presentations and severity due to its multisystem involvement and reversal of viscera orientation. There are currently no reliable noninvasive diagnostic methods for this disease and the correct diagnosis is often delayed by years, causing irreversible pulmonary damage with subsequent morbidity ${ }^{11}$. Lundberg et al. conducted intranasal nitric oxide inhalation test on diseased and control subjects. There was a $98 \%$ reduction of level of nasal nitric oxide concentration in patients with Kartagener syndrome compared to age-matched controls suggesting that nitric oxide measurements (which are noninvasive and can easily be performed even in infants) could be of help in the early diagnosis and hence management of the disease process ${ }^{12}$.

Early diagnosis and treatment is important to prevent long term sequel and morbidity associated with it. Genetic counseling, social, psychological and fertility issues should be addressed once it is diagnosed and help these patients to live with Kartagener syndrome without morbidity and in a dignified way.

Differential diagnosis is from cystic fibrosis (an inherited disorder), allergic bronchopulmonary aspergillosis, a fungal infection and alpha-1-antitrypsin deficiency, an inherited disease seen in white population ${ }^{13}$.

Kartagener syndrome should be kept in mind in a patient presenting with:

1. Recurrent sinusitis and bronchiectasis.

2. Asthma like symptoms and signs responding poorly to conventional treatment.

3. Recurrent lower respiratory tract infections causing fever, sweating and weight loss; tempting the physician to give a trial of antituberculous drugs.

From the preceding discussion, it is also clear that those patients with Kartagener syndrome having situs inversus totalis will present with left sided appendicitis if they develop this problem at some stage in their lives ${ }^{14}$.

TEACHING POINT: Kartagener syndrome is subset of primary ciliary dyskinesia, in which there is an ultrastructural defect in formation of cilia leading to poor mucociliary clearance. It consists of a triad of bronchiectasis, situs inversus and features of chronic sinusitis. Early diagnosis and treatment of this syndrome is important to prevent the deterioration of the lung function and the morbidity caused due to severe intractable upper and lower respiratory tract infections.

\section{ABBREVIATIONS}

PCD = Primary Ciliary Dyskinesia

$\mathrm{CT}=$ Computed Tomography

\section{REFERENCES}

1. AS Dabhi, SR Chaudhari, PB Thorat, HB Pandya, MC Shah, UN Meswani, PR Vaghela. Kartagener's Syndrome:A Triad of Bronchiectasis, Situs Inversus, and Chronic Sinusitis. JIACM 2005; 6(3): 241-3

2. Shakya K. Kartagener Syndrome:A Rare Genetic Disorder.J Nepal Med Assoc.2009; 48(173):62-5 PMID:19529061 
3. Siewert A. Uber einen Fall von Bronchiektasie bei einem Patientem mit Situs inversus viscerum. Berlin Klin Wochenschr 1904; 41:139-41.

4. Braunwald,Fauci AS, Kasper DL et al. Bronchiectasis. Harrison's principles of internal medicine. New York 2004; 2:1541-3.

5. Pifferi M, Cangiotti AM, Ragazzo V et al. Primary ciliary dyskinesia: diagnosis in children with inconclusive ultrastructural evaluation. Pediatr Allergy Immunol 2001; 12:274-82 PMID:11737674

6. Blouin JL, Meeks M, Radhakrishna U, Sainsbury AJ, Gehring C, Duriaux Saïl G et al. Primary ciliary dyskinesia: a genome-wide linkage analysis reveals extensive locus heterogeneity. Eur J Hum Genet 2000 Feb; 8(2):109-118. PMID:10757642

7. King SM.The dynein microtubule motor. Biochim Biophys Acta 2000; 1496: 60-75. PMID:10722877

8. Afzelius BA, Mossberg B. Immotile cilia syndrome, including Kartagener syndrome. In: Scriver CR et al., (editor).The Metabolic Basis of Inherited Disease, 6th Ed. New York :McGraw-Hill, 1989.

9. Bent JP, Olearczyk M. Kartagener Syndrome. Emedicine [Internet]. Jan 3, 2007[cited June3,2008].

10. Marcus P.Kennedy, Peadar G. Noone, Margaret W.Leigh,Maimoona A. Zariwala, Susan L.Minnix, Michael R. Knowles,Paul L. High-Resolution CT of Patients with Primary Ciliary Dyskinesia. AJR 2007;188:1232-38 PMID:17449765

11. Levison H, Minsdorff CM, Chao J, et al. Pathophysiology of the ciliary motility syndromes.Eur J Respir Dis 1983; 64 (Suppl. 127): 102-116. PMID:6225657

12. Lundberg JON, Weitzberg E, Nordvall SL, Kuylenstierna R, Lundberg JM,Alving K. Primarily nasal origin of exhaled Nitric Oxide and absence in Kartagener syndrome. Eur Respir J 1994; 7: 1501-04. PMID:7957837

13. Campos MA, Wanner A, Zhang G, Sandhaus RA. Trends in the diagnosis of symptomatic patients with alpha1-antitrypsin deficiency between 1968 and 2003. Chest. Sep 2005;128(3):1179-86.

14. Kartagener Syndrome. Ihsan-ullah Mahsud and Shahab-ud Din. Gomal Journal of Medical Sciences; July-Dec 2006, Vol. 4, No. 2

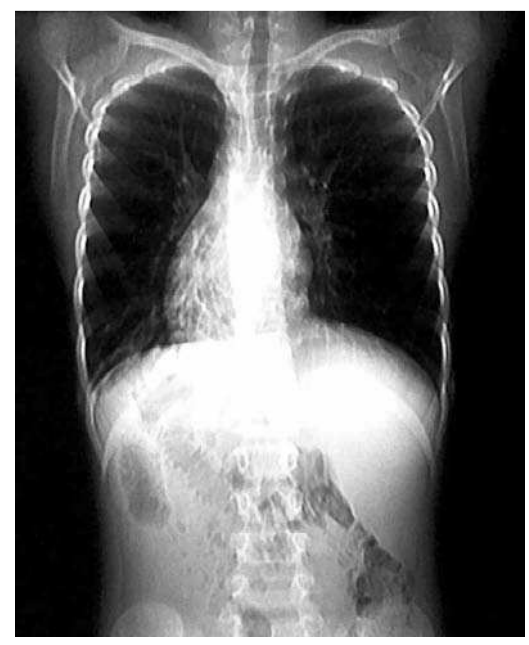

Figure 1. Posteroanterior chest radiograph demonstrates apex of heart on the right side, liver shadow in left hypochondrium and stomach, splenic flexure and spleen on the right side suggestive of situs inversus totalis. 


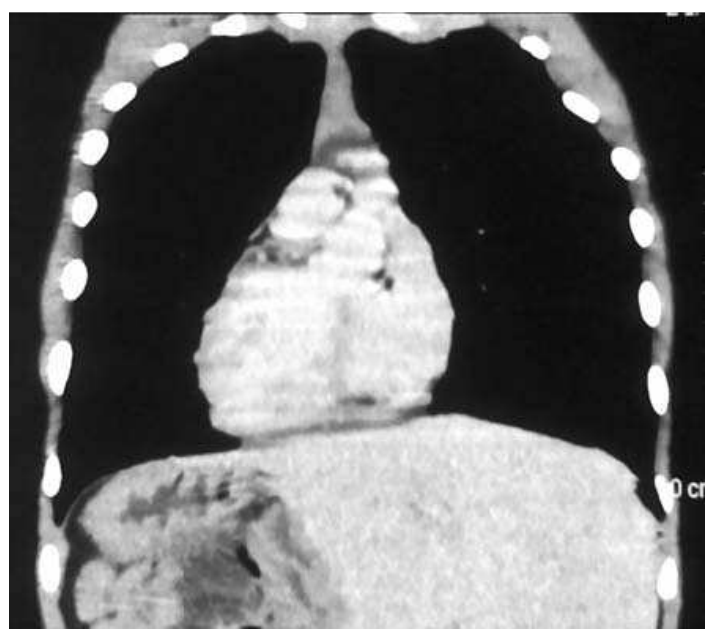

Figure 2. Contrast Enhanced CT Chest and upper abdomen with Coronal Reformat shows the apex of heart on the right side suggestive of dextrocardia with liver on the left side and gastric fundus on right side (Situs Inversus Totalis)

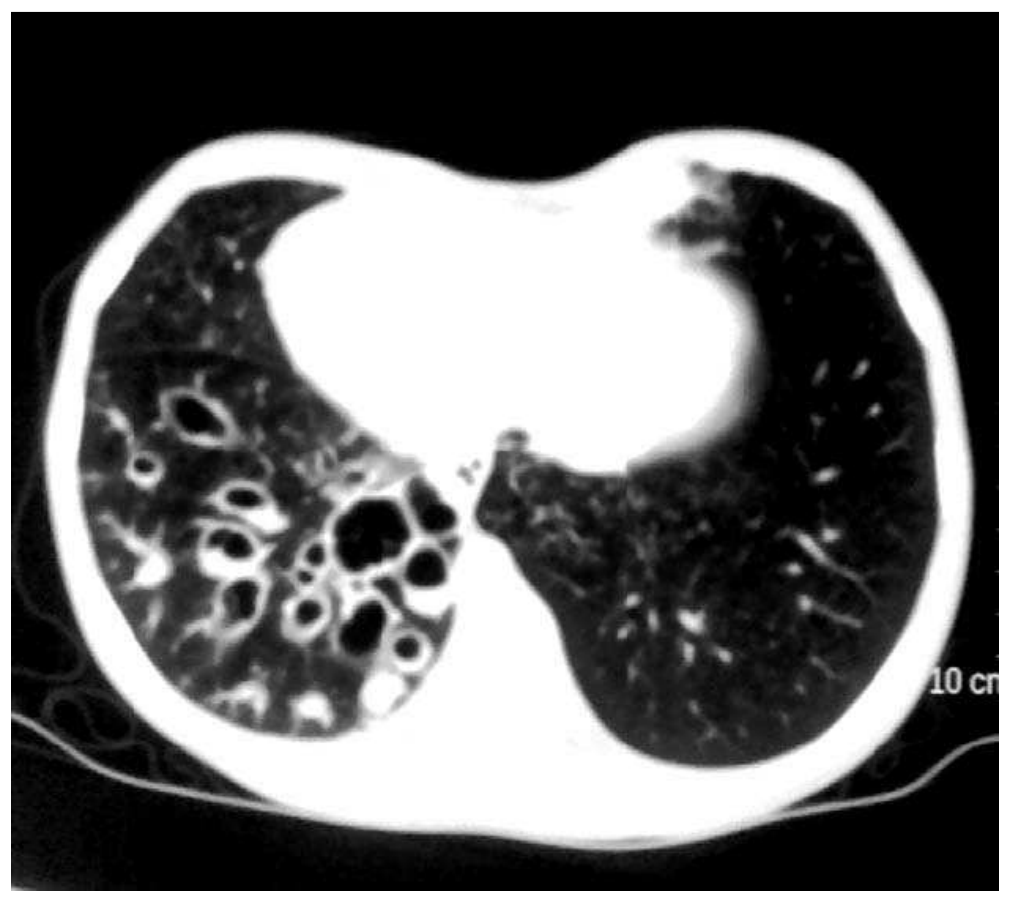

Figure 3. HRCT Scan of Chest showing cystic lesions of variable sizes in the lower lobe of right lung suggestive of central type of bronchiectasis with peribronchial thickening. Air fluid levels are seen in a few of these dilated cystic lesions suggestive of superadded infection. 


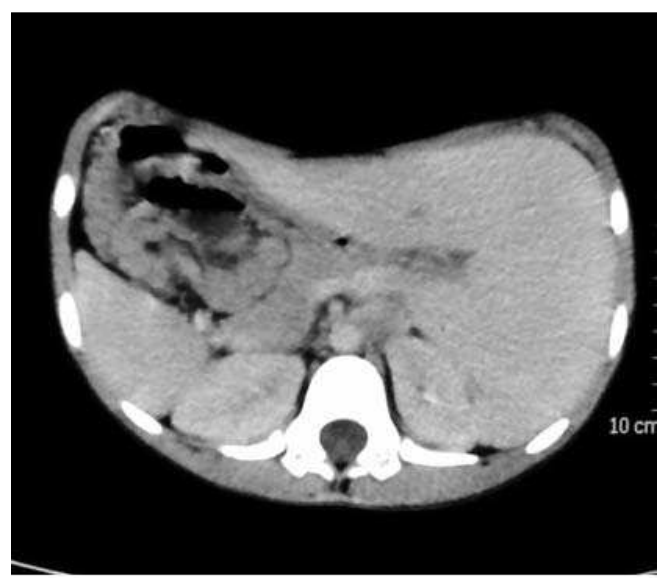

Figure 4. Contrast Enhanced Axial CT Scan of the upper abdomen showing liver on the left side and spleen, stomach and splenic flexure on right side suggestive of Situs Inversus Totalis.

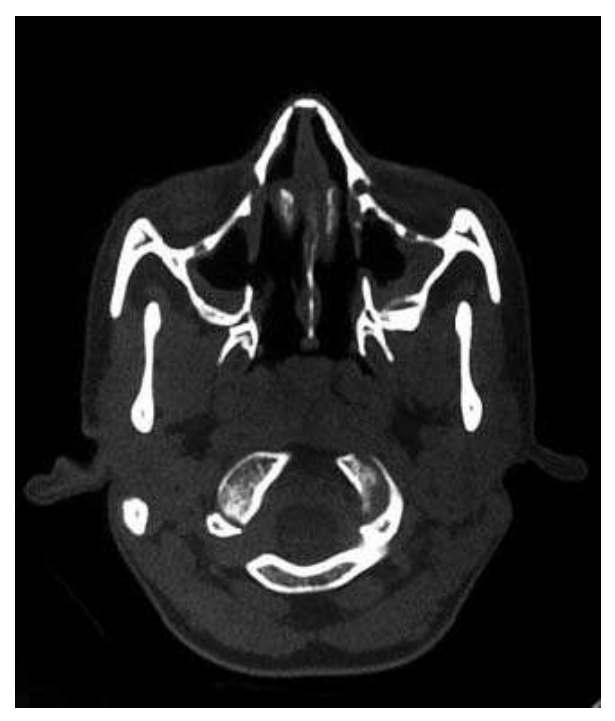

Figure 5. Non Contrast Axial CT scan of the paranasal sinuses of eleven years old child showing the concentric type of mucosal hypertrophy in both maxillary antra Left $>$ right with bilateral inferior turbinate hypertrophy suggestive of rhino sinusitis. 